\title{
MEK/ERK signaling is a critical mediator for integrin-induced cell scattering in highly metastatic hepatocellular carcinoma cells
}

\author{
Nobuyuki Honma ${ }^{1}$, Takuya Genda ${ }^{1}$, Yasunobu Matsuda ${ }^{1}$, Satoshi Yamagiwa ${ }^{1}$, \\ Masaaki Takamura ${ }^{1}$, Takafumi Ichida ${ }^{2}$ and Yutaka Aoyagi ${ }^{1}$ \\ ${ }^{1}$ Division of Gastroenterology and Hepatology, Department of Cellular Function, Niigata University Graduate \\ School of Medical and Dental Science, Niigata City, Japan and ${ }^{2}$ Department of Gastroenterology, Juntendo \\ University School of Medicine and Juntendo University Hospital of Shizuoka, Shizuoka, Japan
}

\begin{abstract}
The human hepatocellular carcinoma (HCC)-derived cell line KYN-2 is thought to provide a good model for studying the molecular basis of invasion and metastasis of human HCC, because it often shows cell scattering in vitro and intrahepatic metastasis in vivo. We previously found that integrin-mediated extracellular signals inactivated E-cadherin in KYN-2, and caused loss of cell-cell contact with gain of cell motility, which is considered to be a critical step in the process of cancer cell invasion and metastasis. To further understand molecular mechanisms involved in biological aggressiveness of HCC, we investigated intracellular signaling involved in integrin-mediated scattering of $\mathrm{KYN}-2$ cells. Cultured $\mathrm{KYN}-2$ cells formed trabecular aggregates in suspension, but when adhering to integrin-stimulating substrata, they scattered according to phosphorylation of extracellular signal-regulated kinase (ERK). Upon treatment with ERK kinase (MEK) inhibitor PD98059, adhered KYN-2 cell scattering was inhibited, tight cell-to-cell contact was recovered, and both E-cadherin and actin filaments accumulated in the area of intercellular contact zone. In contrast, constitutively active MEK1transfected KYN-2 cells showed reduced E-cadherin and actin filaments in the intercellular contact zone, showing a flattened phenotype with broad lamellipodia. Enforced signaling of MEK-ERK pathway in KYN-2 cells suppressed cadherin-mediated homotypic adhesion and increased the potential of cell motility. An antibodybased protein microarray analysis revealed that the cytoplasmic protein $\mathrm{c}-\mathrm{Cbl}$ was significantly downregulated in MEK1-transfected KYN-2 cells, suggesting that $\mathrm{c}-\mathrm{Cbl}$ might be a candidate downstream mediator of integrin/ MEK/ERK-mediated cell scattering. In conclusion, cell scattering of the highly metastatic cell line KYN-2 is regulated through the integrin-MEK-ERK signaling cascade, suggesting that this molecular pathway may be critical in intrahepatic metastasis of human HCC.
\end{abstract}

Laboratory Investigation (2006) 86, 687-696. doi:10.1038/labinvest.3700427; published online 8 May 2006

Keywords: actin; c-Cbl; cell motility; E-cadherin; intrahepatic metastasis; liver cancer

Hepatocellular carcinoma (HCC) is one of the most aggressive malignancies found worldwide. Despite considerable progress in diagnostic and therapeutic modalities, prognosis of HCC remains poor, because it often reoccurs and spreads by metastasis within a short time. ${ }^{1,2}$ HCC metastasis is mainly due to tumor cell dispersal to surrounding liver tissues through the portal vein, ${ }^{3}$ a mechanism which is generally clinically referred to as 'intrahepatic metastasis'. Pathological studies have suggested that cancer cell scattering from the tumor periphery may be closely

Correspondence: Dr T Genda, MD, Division of Gastroenterology and Hepatology, Department of Cellular Function, Niigata University Graduate School of Medical and Dental Science, Asahimachi-dori 1-757, Niigata City 951-8510, Japan.

E-mail: tgenda@sbthp.jp

Received 4 October 2005; revised 13 March 2006; accepted 14 March 2006; published online 8 May 2006 associated with intrahepatic metastasis of HCC, ${ }^{4}$ but its biological significance is not fully understood. In a previous study, we injected various types of human hepatoma cell lines into the livers of SCID mice, and found that their scattering abilities in vitro strongly correlated with potential of intrahepatic metastasis. ${ }^{5}$ When scattering ability of highly-metastatic hepatoma cell lines was inhibited by dominant-negative Rho-kinase or Rho-kinase inhibitors, significant loss in metastatic potential was observed. ${ }^{5,6}$ Taken together, scattering ability of hepatoma cells in vitro might be tightly correlated with their metastatic ability in vivo, and investigation of molecular mechanisms of hepatoma cell scattering seems valuable for understanding the invasive phenotype of HCC.

Among hepatoma cell lines, the human HCCderived cell line KYN-2 has been regarded as a good 
model for investigating the molecular basis of intrahepatic metastasis of HCCs, because it exerts a strong metastatic potential in vivo, with marked cell scattering in vitro compared to other hepatomas. ${ }^{5,7}$ In a previous study, we found that in vitro culture of KYN-2 cells on integrin-stimulating cell-substrata significantly induced cell scattering, and the disruption of E-cadherin-mediated intercellular adhesion, suggesting that one of the main reason for invasive property of HCC is integrin signaling. ${ }^{7}$ Integrin receptors are composed of $\alpha-\beta$ heterodimers that recognize extracellular matrices, which not only provide a link with the actin cytoskeleton, but also serve as signaling receptors affecting cell behavior and various types of gene expression. ${ }^{8}$ It is widely accepted that altered levels in integrin expression are closely involved in gain of tumorigenesis and metastatic ability in cancer cells. ${ }^{9-11}$ In human HCC, reduced expression of $\beta$ integrin subunits $\alpha 2, \alpha 3$ and $\alpha 5$ and overexpression of subunit $\alpha 6$ were reported to be associated with increased aggressiveness. ${ }^{12-14}$ Moreover, several in vitro experiments indicated that $\beta 1$ integrin plays a critical role in the invasive phenotype of HCC cell lines. ${ }^{15,16}$ Therefore, to understand the molecular basis of integrin signaling pathway in HCC, investigating its downstream intracellular signaling seems important. To date, however, there have been no reports, which examined intracellular signaling in HCC with regards to integrin-mediated invasive property.

It has been revealed that integrin-mediated extracellular signals stimulate a wide variety of intracellular signaling events including tyrosine phosphorylation, ${ }^{17,18}$ calcium influx, ${ }^{19}$ and activation of the mitogen-activated protein kinase family such as extracellular signal-regulated kinase (ERK) ${ }^{20}$ and c-Jun NH2-terminal kinase. ${ }^{21}$ Among these intracellular events, ERK signaling seems of value for investigation, because it was reported that activated ERK was frequently observed in human HCC tissues. ${ }^{22}$ Although there have been no reports which examined the molecular relationship between ERK signaling and gain of invasive phenotype of HCC, it is worth noting that in vitro experiments had revealed that the ERK signaling pathway was involved in the migration of pancreatic cancer cells. ${ }^{23}$ Therefore, to further understand the molecular mechanisms of intrahepatic metastasis of HCC, we investigated the biological potential of ERK signaling during integrin-stimulated cell scattering, using the highly metastatic HCC cell line KYN-2.

\section{Materials and methods}

\section{Antibodies and Reagents}

Mouse monoclonal antiphospho-p44/42 ERK1/2 (Thr202/Tyr204) E10 was purchased from Cell Signaling Technology (Beverly, MA, USA), and functional neutralizing mouse monoclonal antibody against $\beta_{1}$ integrin (P4C10) was obtained from Chemicon (Temecula, CA, USA). Rabbit polyclonal antihemagglutinin (HA) tag was from Clonetech (Palo Alto, CA, USA). Mouse monoclonal anti-ERK kinase (MEK) 1, anti-ERK1, anti-ERK2, and anti-cCbl were from Transduction Laboratories (Lexington, KY, USA). Mouse monoclonal anti-E-cadherin (HECD1) was from TaKaRa Shuzo (Siga, Japan). Mouse monoclonal anti- $\beta$ actin (AC-15), polyhydroxyethylmethacrylate (poly-HEMA), poly-L-lysine (PLL) and tetramethyl rhodamine iso thiocyanate (TRITC)-labeled phalloidin were from Sigma Chemical (St Louis, MO, USA). MEK inhibitor PD98059 was from Upstate Biotechnology Inc. (Lake Placid, NY, USA), and the other MEK inhibitor U0126 was from Promega (Madison, WI, USA). Recombinant human epidermal growth factor (EGF) and hepatocyte growth factor (HGF) were from TOYOBO (Osaka, Japan).

\section{Cell Culture}

Human HCC cell line KYN-2 was kindly provided by $\mathrm{Dr} M$ Kojiro (Kurume University, Kurume, Japan). ${ }^{24}$ To evaluate morphological changes of KYN-2 in response to cell-substratum adhesion, cells were cultured on the culture dishes with or without poly-HEMA-coating in RPMI-1640 medium containing antibiotics $(100 \mathrm{U} / \mathrm{ml}$ penicillin and $100 \mu \mathrm{g} / \mathrm{ml}$ streptomycin) with or without $10 \%(\mathrm{v} / \mathrm{v})$ fetal bovine serum. The culture dishes were made of anionic and hydrophilic polystyrene, which enables maintenance of cell adhesion through facilitating matrix deposition when serum is added to the culture media. Poly-HEMA is a nonionic material that blocks extracellular matrix deposition, and cell attachment irrespective of the presence of serum in the media. To investigate effects of growth factors or integrin/MEK signaling effectors on morphological changes in KYN-2 cells, cells were deprived of serum for $12 \mathrm{~h}$ to avoid serum-associated nonspecific stimulation; and then, cells were incubated with appropriate agents with the same media. EGF or HGF was added to the culture medium at a final concentration of $50 \mathrm{or} 40 \mathrm{ng} / \mathrm{ml}$, respectively; and a neutralizing antibody of $\beta_{1}$ integrin P4C10 was added at a concentration of $20 \mu \mathrm{g} / \mathrm{ml}$. MEK inhibitors PD98059 and U0126 were dissolved in dimethylsulfoxide (DMSO), and added to the culture medium at concentrations of 25 and $10 \mu \mathrm{M}$, respectively. After addition of each reagent to the culture medium, cellular morphological changes were observed at regular intervals for $48 \mathrm{~h}$. Cell surface area was quantified by measuring randomly selected individual cells in several scanned photographs.

A calcium-dependent cell dissociation assay was performed as previously described. ${ }^{25}$ Briefly, confluent monolayers were incubated with $0.01 \%(\mathrm{w} / \mathrm{v})$ trypsin with or without $1.25 \mathrm{mM}$ calcium at $37^{\circ} \mathrm{C}$ for $15 \mathrm{~min}$. After addition of trypsin inhibitor, cells 
were gently pipetted 10 times, and degree of cell dissociation was evaluated using a microscope.

\section{Transfection Assay}

MEK1 is known to be activated via phosphorylation of Ser218 and Ser222 residues, and substituting these two sites with aspartic acid can mimic phosphorylation, and lead to a constitutively active type. ${ }^{26,27}$ To activate the MEK/ERK signaling pathway in KYN-2 cells, cells were transfected with MEK1 S218D/S222D mutant-containing pUSE plasmids (Upstate Biotechnology Inc., Lake Placid, NY, USA) using Superfect ${ }^{\mathrm{TM}}$ Transfection Reagents (QIAGEN, Hilden, Germany). Stable transfectants of active-MEK1 plasmids were selected using geneticin (G418; $400 \mu \mathrm{g} / \mathrm{ml}$; GIBCO-BRL), screened for HAtagged MEK1 expression by immunoblotting, and recloned. Control mock transfectants were transfected with empty pUSE plasmid vectors, and cloned in the same manner.

\section{Immunoblotting}

In all experiments, levels of expression and phosphorylation of ERK1 were analyzed $36 \mathrm{~h}$ after plating. Protein samples were extracted in lysis buffer (50 mM Tris $\mathrm{pH}$ 8.0, $150 \mathrm{mM}$ sodium chloride, $1 \mathrm{mM}$ sodium vanadate, $0.1 \%(\mathrm{v} / \mathrm{v})$ sodium dodecyl sulfate, and $1 \%(\mathrm{v} / \mathrm{v})$ Nonidet P-40) for $20 \mathrm{~min}$ at $4^{\circ} \mathrm{C}$. Lysates were precleared by centrifugation, and protein concentration was determined using a Bradford assay (Bio-Rad Laboratories, Hercules, CA, USA). Proteins were electrophoresed on sodium dodecyl sulfate-polyacrylamide gels, and transferred to Immobilon membranes (Millipore, Bedford, MA, USA). After blocking nonspecific sites, filters were reacted with appropriate primary antibodies and corresponding horseradish peroxidase-conjugated secondary antibodies. Peroxidase-labeled bands were visualized using an enhanced chemiluminescence detection system (Amersham International, Buckinghamshire, UK).

\section{Immunocytochemistry}

For immunocytochemical analysis, cells were fixed with $4 \%(\mathrm{v} / \mathrm{v})$ paraformaldehyde in phosphatebuffered saline (pH 7.4) containing $2 \%(\mathrm{w} / \mathrm{v})$ sucrose, and permeabilized with $0.1 \%(\mathrm{v} / \mathrm{v})$ Triton X-100. Cells were incubated with $2 \%(\mathrm{v} / \mathrm{v})$ normal swine serum for blocking nonspecific reaction sites, reacted with appropriate primary antibodies at $4{ }^{\circ} \mathrm{C}$ overnight, followed by incubation with the corresponding FITC-labeled secondary antibodies. Actin filaments were visualized using TRITC-labeled phalloidin. Immunostained cultured cells were mounted using Vectashield (Vector Laboratories, Burlingame, CA, USA), and examined using a Zeiss
LSM410 confocal microscope (Carl Zeiss, Thornwood, NY, USA).

\section{In Vitro Wound Healing Assay}

Cells were plated on six-well plates at a density of $1 \times 10^{6} \mathrm{cells} / \mathrm{plate}$, and cultured overnight in the presence of $10 \%(\mathrm{v} / \mathrm{v})$ fetal bovine serum. When cells were grown to confluence, a linear wound of the monolayer was created by scratching with a pipette tip of a diameter of $1 \mathrm{~mm}$. Morphology and migration of cells were observed, and photographed at regular intervals, and degree of cell motility was assessed as the ratio of the wound width between 0 and $48 \mathrm{~h}$.

\section{Antibody-Based Protein Microarray}

To investigate downstream signaling molecules during integrin/MEK/ERK-mediated cell scattering, an antibody-based microarray assay was performed. Pairs of protein samples were obtained from KYN-2 cells cultured for $36 \mathrm{~h}$ on poly-HEMA and fibronectin-coated dishes, or from MEK2D and mock-transfected KYN-2 cells cultured for $36 \mathrm{~h}$ on uncoated plastic dishes. Cells were processed for Antibody Microarray ${ }^{\mathrm{TM}}$ (BD biosciences Clontech, Palo Alto, CA, USA) to assess expression profiles of 512 molecules (http//www.clontech.co.jp). Each of the paired protein samples was labeled with fluorescent dye Cy3 or Cy5, and was competitively hybridized on the same slide surface where 512 antibodies were spotted. Image analysis was performed using the Axon GenePix 4.1 software package (Axon Instruments, Foster City, CA, USA). Expression ratios of each molecule in the paired samples were normalized using an internal control according to the manufacturer's protocols, and internally normalized ratios $<0.8$ or above 1.8 were considered as being significant among samples.

\section{Results}

\section{Morphological Changes of KYN-2 Cells in Response to} Cell-Substratum Adhesion

When KYN-2 cells were cultured on poly-HEMAcoated dishes in the presence of serum, they did not adhere to the dishes, but floated in media, and tightly aggregated with each other (Figure 1a, upper left). In contrast, when KYN-2 cells were cultured on uncoated plastic dishes in the same conditioned culture media, they adhered on the plates and markedly scattered, showing weavy-protruded cell membranes (Figure 1a, upper right). Immunocytochemical analysis showed that actin filaments of KYN-2 cells plated on poly-HEMA were specifically localized in the area of cell-to-cell attachment like a honeycomb, which was similar to cortical actin bundles in differentiated epithelial cells (Figure 1a, 
lower left). However, in cells adhered to culture dishes, actin filaments were distributed in the cytoplasm, and partially accumulated at the leading edge of ruffled cell membranes forming lamellipodia, which is characteristic of motile cells (Figure 1a, lower right).

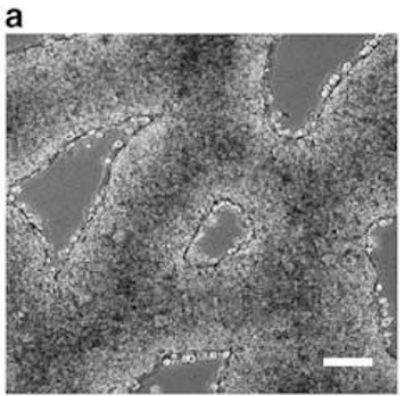

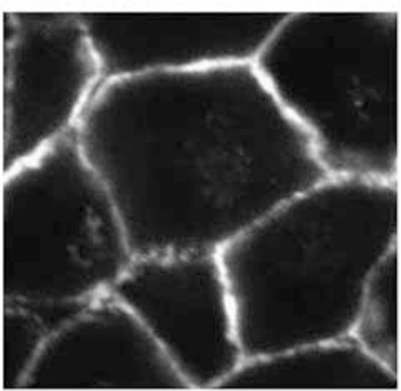

Poly-HEMA
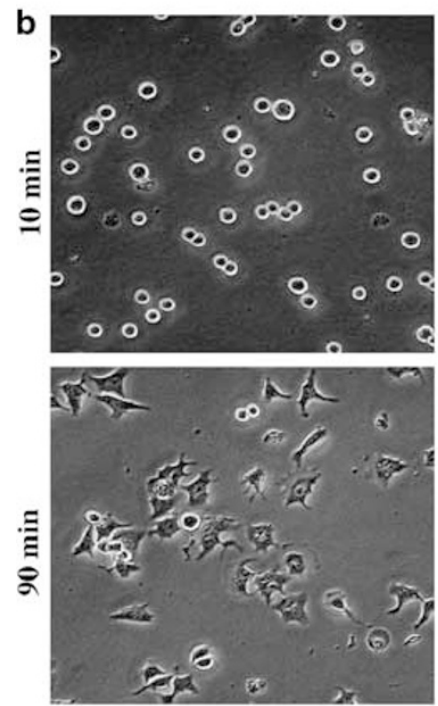

Col 1

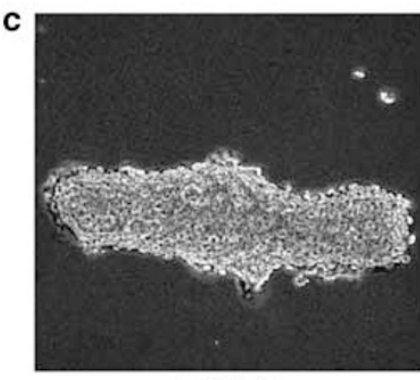

P4C10
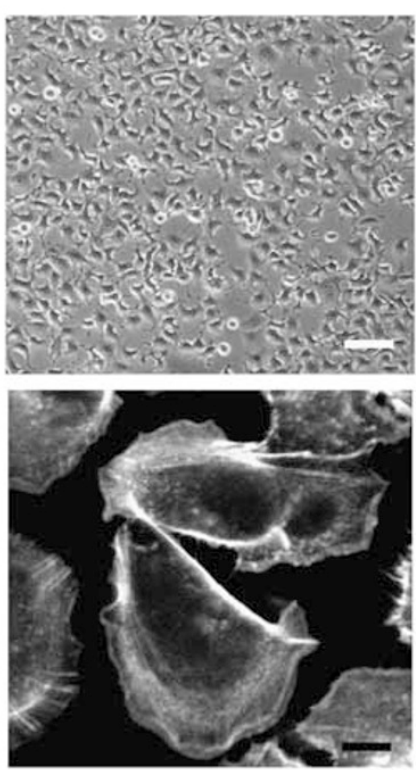

TCD
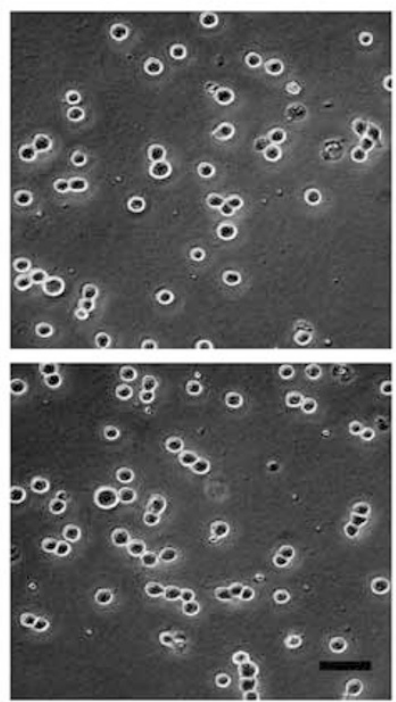

PLL

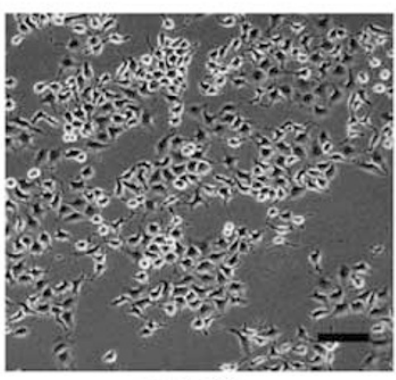

nm IgG

\section{Cell Scattering of KYN-2 Cells is Mediated by Integrin-Stimulating Substratum}

To investigate whether growth factors affected cell scattering in KYN-2 cells, EGF or HGF was added to the serum-free culture medium for blocking nonspecific integrin ligands. Neither EGF nor HGF affected degree of cell scattering in cells plated on uncoated and fibronectin (FN)- or type I collagen (Col I)-coated plastic dishes, indicating little involvement of these growth factors in morphologic changes of KYN-2 cells (data not shown).

To address the roles of integrins in cell-substratum-dependent morphological changes in $\mathrm{KYN}-2$ cells, cells were plated on the dishes coated with either FN, Col I (both are specific ligands of integrins), or PLL (a positively-charged nonspecific adhesion-promoting polypeptide), and cultured in media deprived of serum. When KYN-2 cells were seeded on FN- or Col I-coated dishes, they adhered to plates within $10 \mathrm{~min}$, and scattered over a period of $90 \mathrm{~min}$, showing lamellipodia extension with membrane ruffles (Figure 1b, left column). On the other hand, when cells were seeded on PLL-coated dishes, they adhered to dishes but did not scatter (Figure 1b, right column). Shapes of cells plated on PLL remained unchanged, and lamellipodia extensions or membrane ruffles were never observed. When a neutralizing antibody against $\beta_{1}$ integrin P4C10 was added to the medium, cells plated on FN or Col I-coated dishes significantly aggregated with reduced scattering and decreased lamellipodia (Figure 1c). P4C10 also blocked scattering of cells plated on uncoated plastic dishes (data not shown), indicating that cell scattering of $\mathrm{KYN}-2$ cells is mediated by integrin.

\section{ERK is Phosphorylated through Integrin-Mediated Cell-Substratum Adhesion}

To analyze the status of ERK signaling during integrin-elicited cell scattering, phosphorylated levels of ERK in KYN-2 cells in different conditions were analyzed by immunoblotting. There were no differences in total amounts of ERK1 (Figure 2a, bottom line) and ERK2 (data not shown) between cells plated on poly-HEMA-coated and uncoated

Figure 1 Morphological changes of KYN-2 cells with different substrata. (a) KYN-2 cells were cultured on the dishes with or without poly-HEMA-coating, in the presence of $10 \%(\mathrm{v} / \mathrm{v})$ serum. Morphology was examined by phase-contrast microscopy (upper), and actin filaments were stained with TRITC-phalloidin (lower). (b) KYN-2 cells were cultured on dishes coated with type I collagen (col I) or poly-L-lysine (PLL) without serum, and photographed after 10 and $90 \mathrm{~min}$. (c) Phase-contrast images of KYN-2 cells cultured on fibronectin-coated dishes with neutralizing monoclonal antibody against $\beta 1$ integrin (P4C10, right) and nonimmunized mouse IgG (nmIgG, left). Scale bars: $100 \mu \mathrm{m}$ (a, upper, b and $\mathbf{c}$ ), $5 \mu \mathrm{m}$ (a, lower). 
plastic dishes, in the presence of serum. However, phospho-ERK1/2 was markedly elevated in cells adhered to uncoated plastic dishes compared to cells cultured on poly-HEMA-coated dishes (Figure 2a, upper line). Increased levels of phospho-ERK1/2 were also observed in cells plated on FN- or Col Icoated plates, immediately after attachment to dishes (Figure 2b). However, cells plated on PLL did not show any phosphorylated ERK1/2 during culture maintenance (Figure 2b). P4C10 treatment significantly inhibited ERK1/2 phosphorylation in cells adhered to FN-coated plates (Figure 2c), indicating that blockade of $\beta 1$ integrin signaling prevented ERK phosphorylation.

a
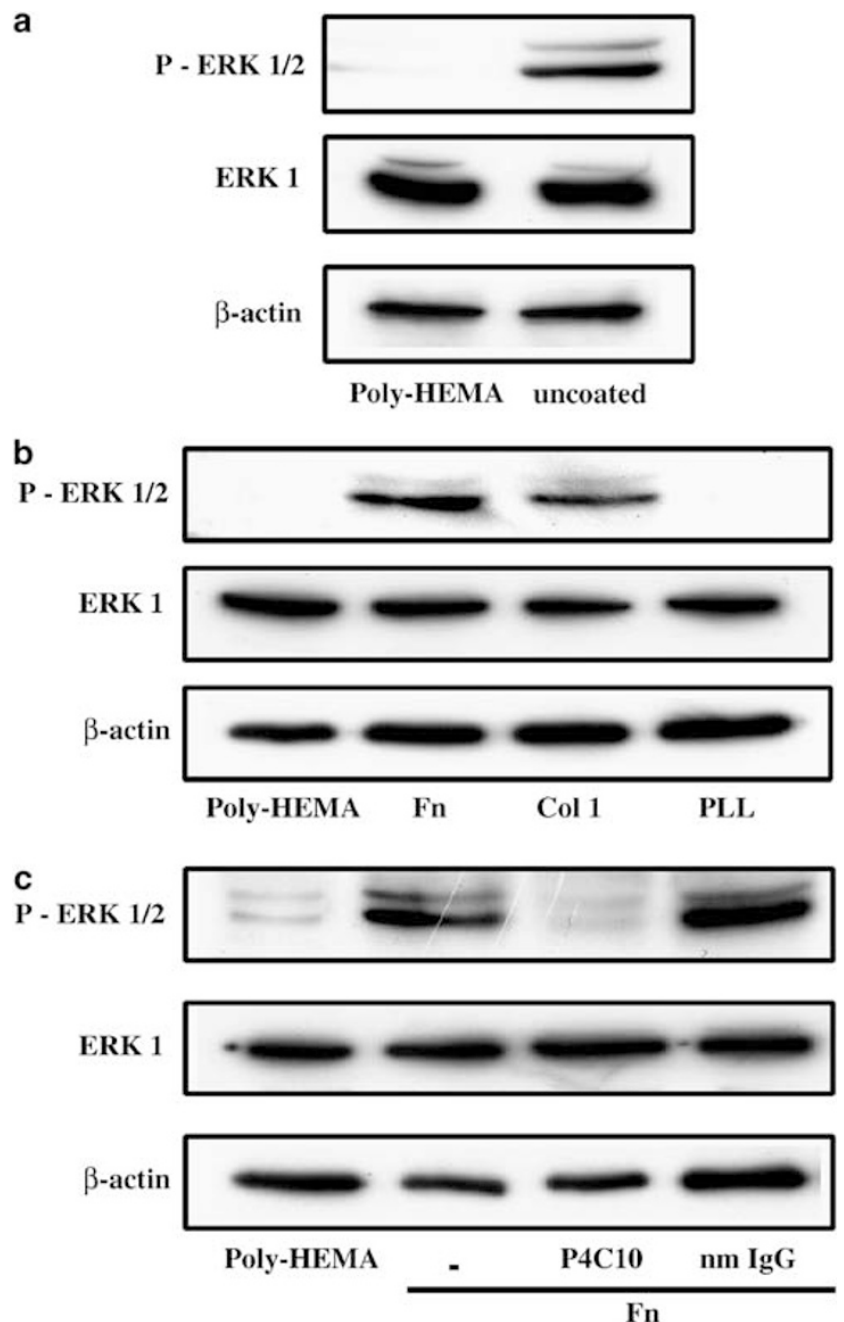

Figure 2 Immunoblotting of total and phosphorylated ERK. (a) KYN-2 cells were cultured on poly-HEMA coated dishes (polyHEMA) or uncoated plastic dishes (uncoated), in the presence of $10 \%(\mathrm{v} / \mathrm{v})$ serum. (b) Cells were cultured on dishes coated with poly-HEMA (poly-HEMA), fibronectin (Fn), type I collagen (I col) or poly-L-lysine (PLL) in serum-deprived medium. (c) Cells were cultured on dishes coated with poly-HEMA (poly-HEMA) or fibronectin ( $\mathrm{Fn})$, in the presence of nonimmunized mouse IgG (nmIgG) or neutralizing monoclonal antibody against $\beta 1$ integrin (P4C10).

\section{Blockade of MEK-ERK Signaling Inhibits Cell Scattering of KYN-2 Cells}

It is well known that activity of ERK is directly regulated by its upstream signaling mediator MEK. ${ }^{28}$ Therefore, to comprehensively analyze the involvement of MEK-ERK signals in integrin-elicited cell scattering, MEK inhibitor PD98059 was added to KYN-2 cells plated on uncoated plastic dishes, in the presence of serum. Immunoblot analysis showed that levels of ERK1/2 phosphorylation were significantly reduced after treatment with PD98059, confirming that blockade of MEK signaling prevents ERK activity in KYN-2 cells (Figure 3a). Under phase-contrast microscopic examination, control DMSO-treated cells appeared to be of membraneruffled fusiform shape without tight cell-to-cell contact (Figure 3b, left upper column), which was the same as cells cultured on uncoated plastic dishes, as shown in Figure 1a. After treatment with PD98059 for $12 \mathrm{~h}$, cells changed into a polygonal shape, decreased membrane ruffles, recovered tight cell-cell contact, and formed cohesive monolayer colonies (Figure 3b, right upper column). In control DMSO-treated cells, E-cadherin was diffusely expressed in the cytoplasm and faintly on cell membranes, but in PD98059-treated cells it was limited along with cell-cell contact areas (Figure 3b, middle column). Actin filaments were distributed in the cytoplasm, and accumulated at the ruffled cell membranes in DMSO-treated cells, whereas in PD98059-treated cells, they were mainly localized in the area of cell-to-cell attachment like cortical bundles (Figure $3 \mathrm{~b}$, bottom column).

\section{MEK-ERK Signaling Cascade Induces Cell Spreading of KYN-2 Cells}

To further gain insight into the roles of the MEKERK signaling cascade, we manipulated MEK1 activity in KYN-2 cells. Three clones of constitutively active MEK1 transfectants MEK2D-6, -8, -9, and two mock transfectants Mock 1 and 2 were obtained by stable transfection, and immunoblot analysis showed that all three MEK2D clones expressed an epitope-tagged active form of MEK1 (Figure 4, top column). All MEK2D clones showed significantly higher levels of phosphorylated ERK1/ 2 compared to those from the parent and mock transfectants, confirming that ERK was constitutively activated by MEK1 signaling in these clones (Figure 4). We first investigated whether enhanced MEK-ERK signaling affected cell shape of KYN-2 cells compared to cells plated on FN or Col I plates in our study. To avoid interference in cell shape and width by mutual neighboring cells, microscopic examination was performed at the time when cells were grown to sub-confluent densities. All three MEK2D clones cultured on uncoated plastic dishes showed broad lamellipodia with ruffling at edges of cell membranes, and exhibited marked somal 
692

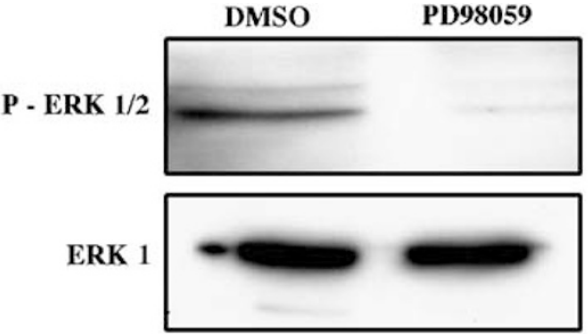

b
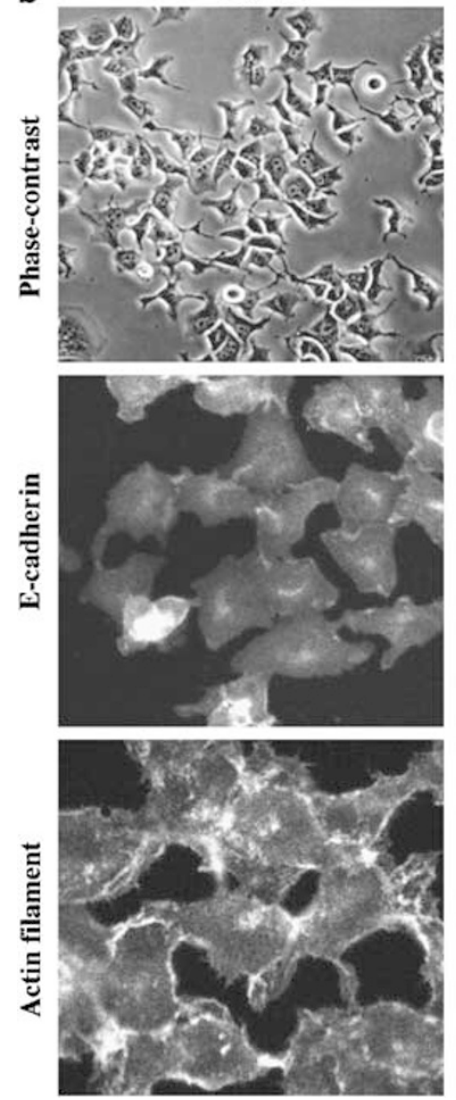

Figure 3 Effects of the MEK inhibitor PD98059 on KYN-2 cells. Cells were cultured on uncoated plastic dishes for $12 \mathrm{~h}$ in media containing $25 \mu \mathrm{M}$ PD98059 dissolved in $0.002 \%(\mathrm{v} / \mathrm{v})$ DMSO. Control cells were cultured with DMSO only. (a) Expressions of ERK1 and phosphorylated ERK1/2 in each sample were analyzed by immunoblotting. (b) Phase-contrast images (upper), confocal fluorescent images of E-cadherin (middle) and actin filaments (lower) in DMSO- or PDD98059-treated KYN-2 cells are shown. Scale bars: $20 \mu \mathrm{m}$ (b, upper) $10 \mu \mathrm{m}$ (b, middle and lower).

flattening compared to parent and mock transfectants (Figure 5a). Mean cell surface area in MEK2D was 1.8- to two-folds of that of parent cells and mock transfectants (Figure 5b), indicating that MEK activation specifically induced spreading phenotype.

\section{MEK1 Activation Inhibits E-Cadherin-Mediated Cell-to-Cell Attachment}

As we previously found that integrin-mediated spreading and migration of KYN-2 cells coincided with E-cadherin inactivation, ${ }^{7}$ we examined

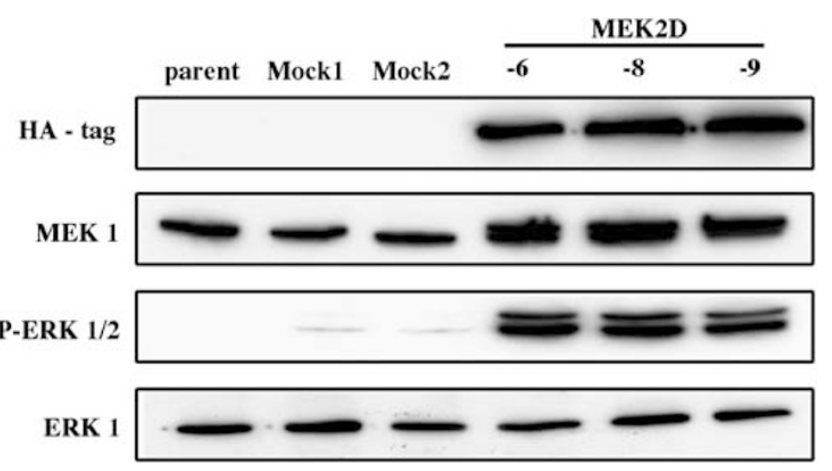

Figure 4 Immunoblotting of MEK1 in transfectants. Expressions of HA-tag, MEK1, phosphorylated ERK1/2 and ERK1 in each cell lysate of parent KYN-2 cells (parent), two control clones (mock1 and 2), and three clones expressing the constitutively active MEK1 mutant (MEK2D-6, -8, and -9) were analyzed by immunoblotting.
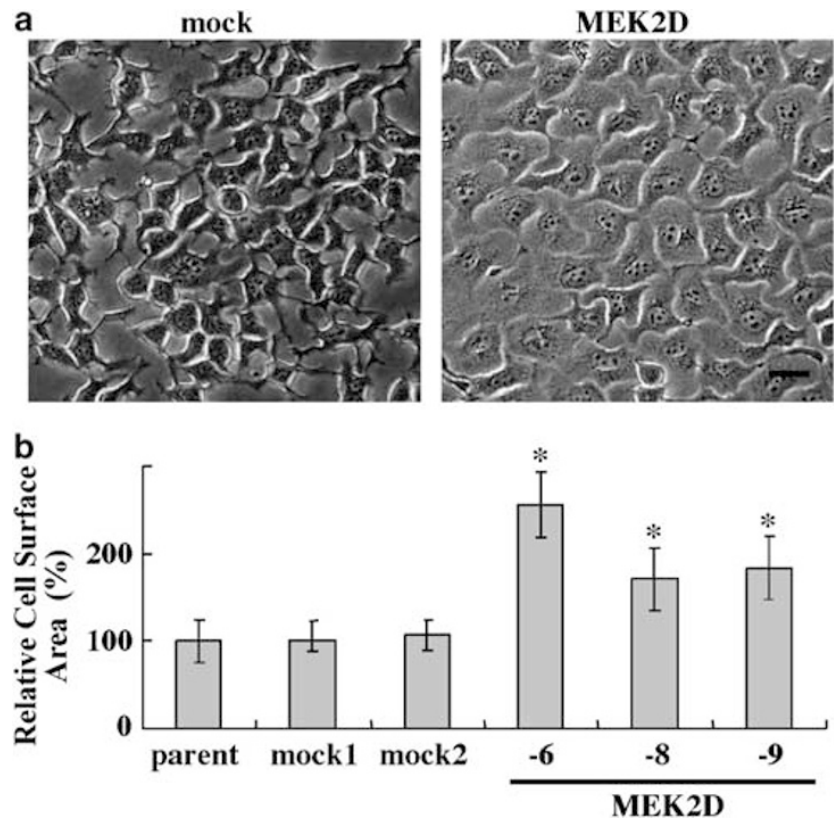

Figure 5 Morphological features of active MEK1 transfectants. (a) Phase-contrast view of mock cells (mock) and MEK2D clone 6 (MEK2D). Both cells were cultured in subconfluent densities on uncoated plastic dishes in the presence of $10 \%(\mathrm{v} / \mathrm{v})$ serum. (b) Cell surface area was quantified by scanning photographic images of 100 individual randomly-selected cells. Each bar shows the ratio of the cell surface area of cells compared to those of parent cells. Data represent means \pm s.d. $(P<0.0001$; an asterisk shows significance as assessed by Student's $t$-test). Scale bar: $20 \mu \mathrm{m}$.

whether cadherin-mediated cell-cell adhesion was influenced by MEK-ERK activation. Cells were grown to confluence to analyze the status of Ecadherin and actin filament in the area of cell-cell contact by immunocytochemistry. We found that E-cadherin expression in parent cells and mock transfectants was strong at the site of cell-cell contact, while was weak in MEK2D (Figure 5a, middle). Actin filaments in parent and mock transfectants were prominently localized at cell-cell contact sites like distinct cortical bundles, but were faint in the same area of MEK2D (Figure 5a, lower). 
As E-cadherin expression was found to be weak in the area of cell-cell contact in MEK2D clones, a calcium-dependent dissociation assay was performed to investigate whether MEK-ERK signal influenced E-cadherin-mediated homotypic cell-tocell adhesion. This assay is based on the mechanism that cultured adherent cells lose active cadherin, and are easily dissociated upon removal of calcium during trypsinization, but when calcium is added to the trypsin solution, cells hardly dissociate due to preserved cadherin function. In this assay, parent cells, mock transfectants and all MEK2D transfectants completely dissociated into single cells when calcium was deprived (Figure 6b, upper column). In the presence of calcium, parent cells and mock transfectants hardly dissociated during trypsinization. In contrast, MEK2D transfectants easily dissociated into single cells even in the presence of calcium (Figure 6b, lower column), indicating that enforced MEK-ERK signaling suppressed cadherinmediated homotypic adhesion in KYN-2 cells.

\section{MEK-ERK Signaling Cascade Induces Cell Motility in an In Vitro Wound Assay}

To evaluate functional relationships between MEKERK signaling and cell motility in KYN-2 cells, in vitro wound assays were performed using active MEK1 transfectants. Within $48 \mathrm{~h}$ after scratching confluent cell layers, parent cells and mock transfectants had traversed to some extent but could not completely recover the wound of monolayered cells. However, all three MEK2D clones completely recovered the wound, and re-established a confluent monolayer during the same period (Figure 7a). Mean ratio of wound recovery was $70 \%$ in parent and mock transfectants, and $100 \%$ in MEK2D clones at $48 \mathrm{~h}$, indicating a greater motility of the active MEK1 transfectants (Figure 7b).

\section{C-Cbl is Downregulated during Integrin-MEK-ERK Signal-Induced Cell Scattering}

To investigate mechanisms involved in integrinMEK-ERK-induced hepatoma cell scattering, expression profiles of 512 signaling molecules (transcription factors, signaling mediators, cell cycle regulators, and cytoskeletal components, etc.) were analyzed using an antibody-based protein microarray. In all, 22 molecules showed significantly altered expression levels between KYN-2 cells plated on poly-HEMA-coated dishes and cells plated on fibronectin-plated dishes. Of these, similar expression profiles were observed for a
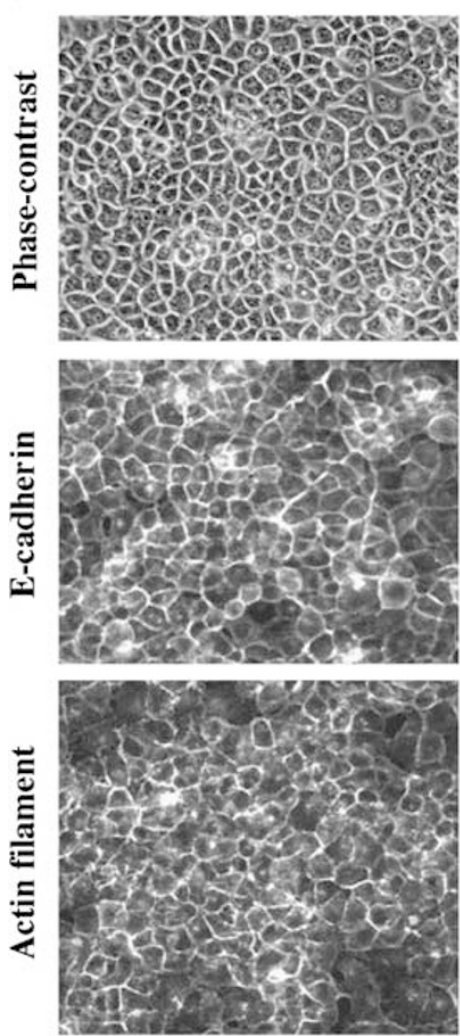

MEK2D
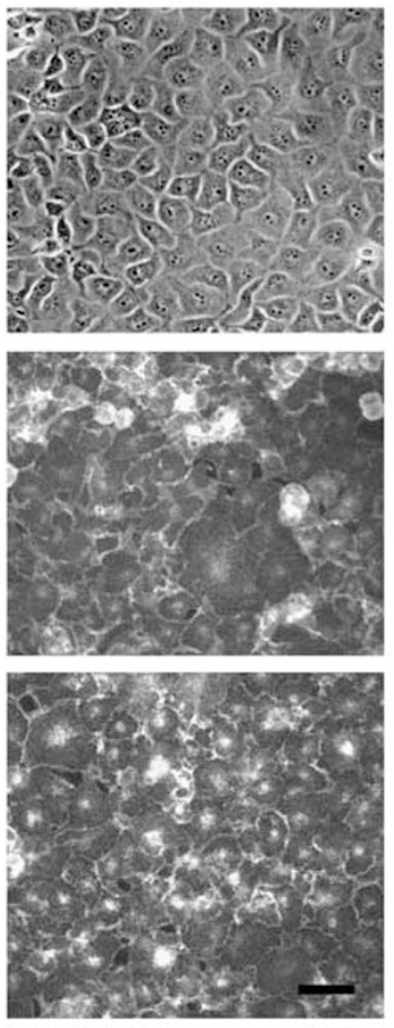

b
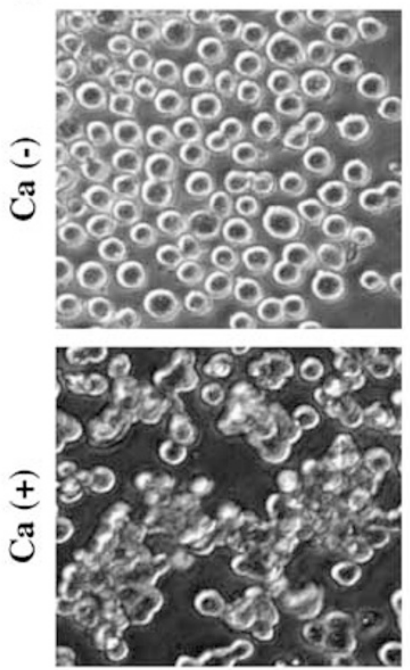

Figure 6 Reduced expression of E-cadherin and actin filaments in the area of cell-to-cell contact in active MEK1 transfectants. (a) Phasecontrast images (upper), confocal fluorescent images of E-cadherin (middle) and actin filaments (lower) of mock cells (left) and MEK2D clone 6 (right) grown to confluence on the uncoated plastic dishes. (b) Confluent cultured monolayer cells were treated with $0.01 \%(\mathrm{w} / \mathrm{v}$ ) trypsin at $37^{\circ} \mathrm{C}$ for $15 \mathrm{~min}$ without (upper) or with (lower) $1.25 \mathrm{mM}$ calcium. Scale bar: $50 \mu \mathrm{m}$. 
694

a
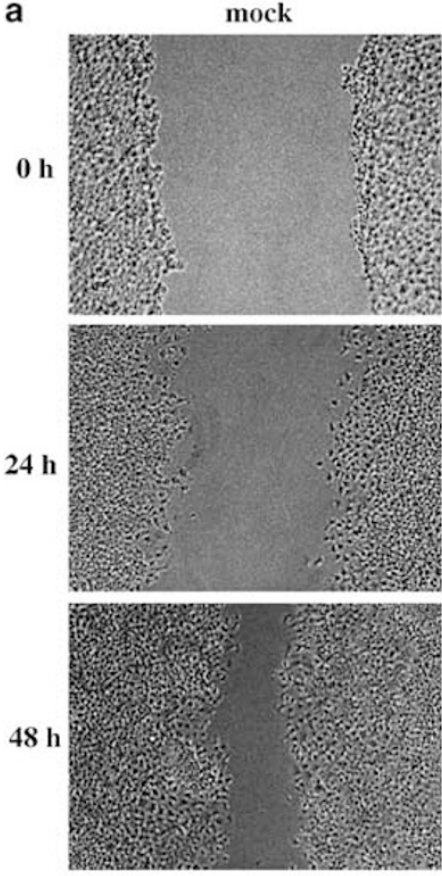

b

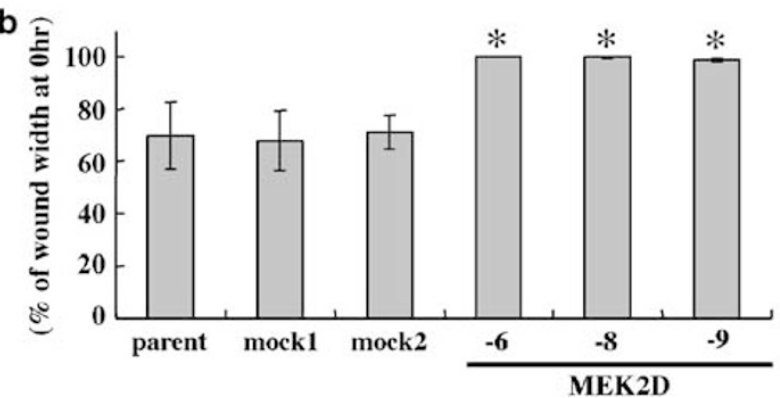

Figure 7 Increased motility of active MEK1 transfectants as assessed by an in vitro wound assay. (a) Phase-contrast images of mock transfectants (mock) and MEK2D clone 6 (MEK2D) are shown. Indicated times $(0,24$, and $48 \mathrm{~h})$ depict the duration after scratching of the monolayer. (b) Each bar shows the ratio of the wound width between before and after $48 \mathrm{~h}$ of scratching. Data represent means \pm s.d. $(P<0.0001$, an asterisk shows significance compared to parent cells using a Student's $t$-test). Three experiments were independently performed in the assay. Scale bar $200 \mu \mathrm{m}$

13 molecules between MEK2D-transfected KYN-2 and mock-transfected cells (Table 1). Among these candidates, immunoblot analysis confirmed that cCbl was significantly downregulated in MEK2D transfectants compared to control mock transfectants and parent cells (Figure 8). Densitometry analysis showed that c-Cbl expression was reduced by about $90 \%$ in MEK2D transfectants compared to controls.

\section{Discussion}

Cancer metastasis is generally considered to be a multistep process, that is, it involves (1) cell detachment and scattering from a primary tumor, (2) migration into the stroma, (3) entry into the
Table 1 Antibody microarray analysis

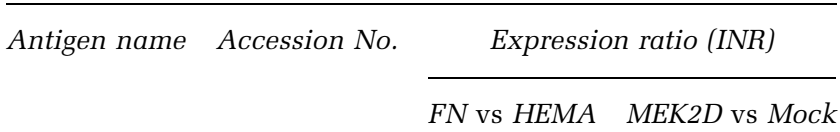

Upregulated by integrin/MEK signaling

CD28

Downregulated by integrin/MEK signaling

$\begin{array}{llll}\text { BTF } & \text { Q9NYF8 } & 0.34 & 0.68\end{array}$

$\begin{array}{llll}\text { NuMA } & \text { Q14980 } & 0.49 & 0.58\end{array}$

$\begin{array}{llll}\text { C-NAPI } & 060588 & 0.38 & 0.40\end{array}$

$\begin{array}{llll}\text { NF-kB } & \text { Q04206 } & 0.51 & 0.51\end{array}$

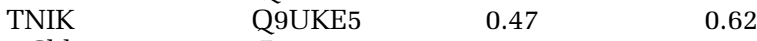

$\begin{array}{llll}\text { c-Cbl } & \text { P22681 } & 0.50 & 0.64\end{array}$

$\begin{array}{llll}\text { Cdk7 } & \text { P50613 } & 0.42 & 0.50\end{array}$

$\begin{array}{llll}\text { Ezrin } & \text { P15311 } & 0.52 & 0.52\end{array}$

$\begin{array}{llll}\text { Pericentrin } & \text { P48725 } & 0.39 & 0.72\end{array}$

$\begin{array}{llll}\text { MONA } & \text { O75791 } & 0.45 & 0.39\end{array}$

$\begin{array}{llll}\text { Perforin } & \text { P14222 } & 0.52 & 0.68\end{array}$

\begin{tabular}{llll} 
BART & Q9Y2Y0 & 0.44 & 0.72 \\
\hline
\end{tabular}

Representative results of antibody-based microarray analysis of paired samples from KYN-2 cells cultured on fibronectin (FN) vs poly-HEMA (HEMA)-coated dishes, and from MEK2D-transfectants (MEK2D) vs mock controls (Mock). The internally normalized ratio (INR) of paired samples was over 1.00 when expression increased in cells plated on FN-coated dishes or in MEK2D transfectants.

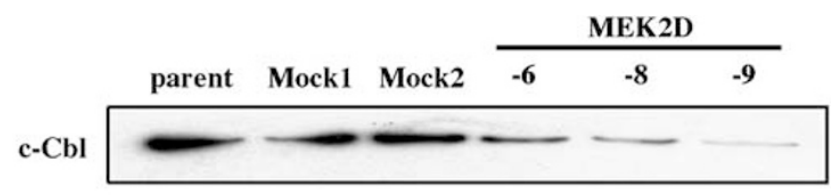

$\beta$-actin

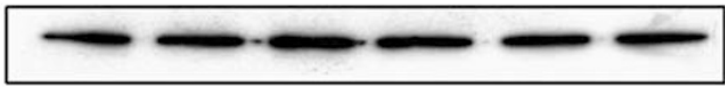

Figure $8 \mathrm{C}$-Cbl expression is downregulated in active MEK1 transfectants. Expressions of $\mathrm{c}-\mathrm{Cbl}$ and $\beta$ actin in $\mathrm{KYN}-2$ parent cells (parent), control clones (mock1 and 2), and clones constitutively expressing active MEK1 mutants (MEK2D-6, -8, and -9) were analyzed by immunoblotting.

vascular and/or lymphatic system, (4) dispersal through the circulation, and (5) proliferation in the seeded organ. Aggressive cancer phenotypes may have properties to complete all these steps, ${ }^{29}$ because each step seems to be a requisite for metastatic behaviors. In HCC, portal invasion and intrahepatic metastasis are frequently observed in cases with infiltrative cancer growth into the surrounding liver at the periphery of tumors, ${ }^{4}$ and gain of migration ability of HCC cells in vitro is considered to reflect their intrahepatic metastatic potential in vivo. ${ }^{5,6}$ Therefore, the initial steps of cancer metastasis including detachment of cancer cells from the primary lesion and scattering to surrounding tissues, may offer the best target to regulate intrahepatic metastatic ability of HCC cells.

To precisely investigate molecular mechanisms of cell-to-cell detachment and scattering of HCC, we used the human HCC-derived cell line KYN-2 which 
has a high propensity for intrahepatic metastasis in vivo and shows high migration ability in vitro. ${ }^{5}$ Our results showed that KYN-2 cells float as trabecular structures with tight cell aggregation when suspended on poly-HEMA-coated dishes. In contrast, when KYN-2 cells were cultured on the dishes in the presence of serum or on collagen type 1 or fibronectin-coated dishes without serum, both of which conditions were under integrin-mediated stimulation, they dissociated from each other, and significantly scattered. These morphological changes were not found in the presence of EGF and HGF, and were effectively inhibited by addition of a neutralizing antibody against $\beta 1$ integrin. Therefore, scattering ability of KYN-2 cells may be exclusively regulated by integrin signaling. In this study, we found a close relationship between intracellular ERK signaling and integrin-elicited morphological changes in this HCC cell line. Immunoblot analysis showed that phosphorylated levels of ERK were significantly increased when KYN-2 cells showed scattering on the dishes with serum or on integrin-stimulating substrata such as collagen type 1 and fibronectin. Conversely, P4C10 treatment prevented phosphorylation of ERK in these cells.

ERK is known to be activated via phosphorylation by MEK signaling. ${ }^{27}$ Therefore, to investigate the role of MEK-ERK signaling cascade in integrinelicited cell scattering and morphological changes in KYN-2, we examined effects of PD98059 which inhibits ERK phosphorylation through the blockade of MEK activity. ${ }^{30}$ Our results showed that PD98059 efficiently blocked cell dissociation, and induced the formation of cohesive colonies of cells plated on the uncoated plastic dishes, in the presence of serum. E-cadherin and actin filaments were predominantly accumulated in the area of cell-cell contact in these cells. Treatment with another MEK inhibitor U0126 resulted in similar results (data not shown), therefore MEK-ERK signaling cascade may be a requisite for integrin-elicited scattering in KYN2 cells. Our in vitro wound assay may support this idea, showing that cell motility increased in active MEK1-transfectants. To further understand the significance of integrin-MEK-ERK signaling in the metastatic potential of hepatoma cells, additional studies using several methodological approaches (eg, in vitro trans-well migration assay, and in vivo mouse model using orthotopic transplantation) should be performed.

In normal epithelial cells, cell-to-cell adhesion is mainly mediated by E-cadherin in a calciumdependent homophilic manner, which is thought to act as an 'invasion suppressor system' in cancer cells. ${ }^{31}$ E-cadherin exerts a signaling linkage signal between extracellular matrices and the actin cytoskeleton through catenins, and promotes cell-to-cell adherence, ${ }^{32}$ thereby preventing cell detachment and scattering. Our present study suggested that MEK-ERK signaling cascade dominated E-cadherin- mediated cell adhesion, as cadherin-mediated homotypic adhesion was abrogated in active MEK1 transfectants in a calcium-dependent cell dissociation assay. A similar finding of the relationship between activated ERK and adherence junction disassembly was recently reported in Ras-transformed or hepatocyte growth factor-stimulated Mardin-Darby canine kidney cells. ${ }^{33}$ However, there have been no studies which examined the correlation of cadherin with integrin-MEK-ERK signaling. To our knowledge, this is the first study to show that integrin-MEK-ERK-mediated cell scattering resists E-cadherin-mediated cell-cell adhesion signal in metastatic cancer cells.

Downstream mechanisms of integrin-MEK-ERKinduced hepatoma cell scattering are intriguing. We analyzed expression profiles in KYN-2 cells using an antibody-based protein microarray, and found that the proto-oncogene product $\mathrm{C}-\mathrm{Cbl}$ was significantly reduced when plated on FN or transfected constitutively active MEK gene. Several studies reported that c-Cbl acted as a negative regulator of cell motility by inhibiting membrane ruffles in NIH 3T3 fibroblasts, ${ }^{34}$ and by ubiquitinating the Rho family guanine nucleotide exchange factor Vav in 293T embryonic kidney epithelial cells. ${ }^{35}$ Our data may provide other insights in the mechanisms of c-Cbl-mediated cell scattering, by showing that c$\mathrm{Cbl}$ was downregulated during integrin/MEK/ERK signaling-induced cell scattering in a highly metastatic hepatoma. It should be noted that c-Cbl acted as a negative regulator of EGF signaling by ubiquitinating EGFR, and inhibition of c-Cbl function is known to result in sustained activation of EGFR/ ERK signaling. ${ }^{36}$ Therefore, together with our data, in a highly metastatic hepatoma $\mathrm{KYN}-2$, downregulation of c-Cbl by integrin/MEK/ERK signaling might result in sustained activation of intrinsic EGFR signaling which enables a positive feedback of EGFR/MEK/ERK signaling to reinforce cell scattering. Further studies should be performed to better understand the biological significance of $\mathrm{c}-\mathrm{Cbl}$ in hepatoma cell scattering.

Recently, a close relationship between MEK-ERK signaling and cell proliferation in HCC tissues was reported. ${ }^{37,38}$ As our data showed that MEK-ERK signaling played a critical role in motility of the invasive HCC cell line KYN-2, this intracellular signaling may not only result in cell proliferation, but also deeply contribute to the first steps of metastasis of HCC. We suggest that blockade of integrin-MEK-ERK pathway may provide a useful therapeutic approach to prevent intrahepatic metastasis of human HCC.

\section{References}

1 Nagao T, Inoue S, Goto S, et al. Hepatic resection for hepatocellular carcinoma. Clinical features and longterm prognosis. Ann Surg 1987;205:33-40. 
2 Nagao T, Inoue S, Yoshimi F, et al. Postoperative recurrence of hepatocellular carcinoma. Ann Surg 1990;211:28-33.

3 Nakashima T. Vascular changes and hemodynamics in hepatocellular carcinoma. In: Okuda K, Peter RL (eds). Hepatocellular Carcinoma. Wiley: New York, 1976, pp 169-203.

4 Kanai T, Hirohashi S, Upton MP, et al. Pathology of small hepatocellular carcinoma. A proposal for a new gross classification. Cancer 1987;60:810-819.

5 Genda T, Sakamoto M, Ichida T, et al. Cell motility mediated by Rho and Rho-associated protein kinase plays a critical role in intrahepatic metastasis of human hepatocellular carcinoma. Hepatology 1999;30: 1027-1036.

6 Takamura M, Sakamoto M, Genda T, et al. Inhibition of intrahepatic metastasis of human hepatocellular carcinoma by Rho-associated protein kinase inhibitor Y27632. Hepatology 2001;33:577-581.

7 Genda T, Sakamoto M, Ichida T, et al. Loss of cell-cell contact is induced by integrin-mediated cell-substratum adhesion in highly motile and highly-metastatic hepatocellular carcinoma cells. Lab Invest 2000;80: 387-394.

8 Juliano RL, Haskill S. Signal transduction from the extracellular matrix. J Cell Biol 1993;120:577-585.

9 Plantefaber LC, Hynes RO. Changes in integrin receptors on oncogenically transformed cells. Cell 1989;56: 281-290.

10 Giancotti FG, Ruoslahti E. Elevated levels of the $\alpha 5 \beta 1$ fibronectin receptor suppress the transformed phenotype of Chinese hamster ovary cells. Cell 1990;60: 849-859.

11 Dedhar S, Saulnier R, Nagle R, et al. Specific alterations in the expression $\alpha 3 \beta 1$ and $\alpha 6 \beta 4$ integrins in highly invasive and metastatic variants of human prostate carcinoma cells selected by in vitro invasion through reconstituted basement membrane. Clin Exp Metastasis 1993;11:391-400.

12 Jaskiewicz K, Chasen MR. Differential expression of transforming growth factor $\alpha$, adhesions molecules and integrins in primary, metastatic liver tumors and liver cirrhosis. Anticancer Res 1995;15:559-562.

13 Yao M, Zhou XD, Zha XL, et al. Expression of the integrin $\alpha 5$ subunit and its mediated cell adhesion in hepatocellular carcinoma. J Cancer Res Clin Oncol 1997;123:435-440.

14 Begum NA, Mori M, Matumata T, et al. Differential display and integrin $\alpha 6$ messenger RNA overexpression in hepetocellular carcinoma. Hepatology 1995;22: 1447-1455.

15 Matsumoto A, Arao S, Otsuki M. Role of $\beta 1$ integrin in adhesion and invasion of hepatocellular carcinoma cells. Hepatology 1999;29:68-74.

16 Carloni V, Mazzocca A, Pantaleo P, et al. The integrin $\alpha 6 \beta 1$ is necessary for the matrix-dependent activation of FAK and MAP kinase and the migration of human hepatocarcinoma cells. Hepatology 2001;34:42-49.

17 Clark EA, Brugge JS. Integrins and signal transduction pathways: the road taken. Science 1995;268:233-239.

18 Schwartz MA, Schaller MD, Ginsberg MH. Integrins: emerging paradigms of signaltransduction. Annu Rev Cell Dev Biol 1995;11:549-599.

19 Schwartz MA. Spreading of human endothelial cells on fibronectin or vitronectin triggers elevation of intracellular free calcium. J Cell Biol 1993;120:10031010.
20 Chen Q, Kinch MS, Lin TH, et al. Integrin-mediated cell adhesion activates mitogen-activated protein kinases. J Biol Chem 1994;269:26602-26605.

21 Miyamoto S, Teramoto $\mathrm{H}$, Cosa OA, et al. Integrin function: molecular hierarchies of cytoskeletal and signaling molecules. J Cell Biol 1995;131:791-805.

22 Ito Y, Sasaki Y, Horimoto M, et al. Activation of mitogen-activated protein kinases/extracellular signalregulated kinases in human hepatocellular carcinoma. Hepatology 1998;27:951-958.

23 Klemke RL, Cai S, Giannini AL, et al. Regulation of cell motility by mitogen-activated protein kinase. J Cell Biol 1997;137:481-492.

24 Yano H, Maruiwa M, Murakami T, et al. A new human pleomorphic hepatocellular carcinoma cell line, KYN-2. Acta Pathol Jpn 1988;38:953-966.

25 Nagafuchi A, Ishihara S, Tsukita S. The roles of catenins in the cadherin-mediated cell adhesion: functional analysis of E-cadherin- $\alpha$ catenin fusion molecules. J Cell Biol 1994;127:235-245.

26 Yan $M$, Templeton DJ. Identification of 2 serine residues of MEK-1 that are differentially phosphorylated during activation by raf and MEK kinase. J Biol Chem 1994;269:19067-19073.

27 Catling AD, Schaeffer HJ, Reuter CW, et al. A prolinerich sequence unique to MEK1 and MEK2 is required for raf binding and regulates MEK function. Mol Cell Biol 1995;15:5214-5225.

28 Crews CM, Alessandrini A, Erkinson RL. The primary structure of MEK, a protein kinase that phosphorylates the ERK gene product. Science 1992;258:478-480.

29 Poste G, Fidler IJ. The pathogenesis of cancer metastasis. Nature 1980;283:139-146.

30 Dudley DT, Pang L, Decker SJ, et al. A synthetic inhibitor of the mitogen-activated protein kinase cascade. Proc Natl Acad Sci USA 1995;92:76867689.

31 Hirohashi S. Inactivation of the E-cadherin-mediated cell adhesion system in human cancers. Am J Pathol 1998;153:333-339.

32 Nagafuchi A, Takeichi M. Cell binding function of E-cadherin is regulated by the cytoplasmic domain. EMBO J 1988;7:3679-3684.

33 Potempa S, Ridley AJ. Activation of both MAP kinase and phosphatidylinositide 3-kinase by Ras is required for hepatocyte growth factor/scatter factor-induced adherens junction disassembly. Mol Biol Cell 1998;9:2185-2200.

34 Scaife RM, Courtneidge SA, Langdon WY. The multi-adaptor proto-oncoprotein $\mathrm{Cbl}$ is a key regulator of Rac and actin assembly. J Cell Sci 2003;116: 463-473.

35 Miura-Shimura U, Duan L, Rao NL, et al. Cbl-mediated ubiquitinylation and negative regulation of Vav. J Biol Chem 2003;278:38495-38504.

$36 \mathrm{Wu}$ WJ, Tu S, Cerione RA. Activated Cdc42 sequesters c-Cbl and prevents EGF receptor degradation. Cell 2003;114:715-725.

37 Huynh H, Nguyen TT, Chow KH, et al. Over-expression of the mitogen-activated protein kinase (MAPK) kinase (MEK)-MAPK in hepatocellular cartcinoma: its role in tumor progression and apoptosis. BMC Gastroenterol 2003;3:19.

38 Tsuboi Y, Ichida T, Sugitani S, et al. Overexpression of extracellular signal-regulated protein kinase (ERK) and its correlation with proliferation in human hepatocellular carcinoma. Liver Int 2004;24:432-436. 